\title{
Supporting Information: A Superconducting Binary Encoder With Multigate Nanowire Cryotrons
}

Kai Zheng ${ }^{1,2,3}$, Qing-Yuan Zhao ${ }^{1,2 *}$, Hai-Yang-Bo Lu ${ }^{1}$, Ling-Dong Kong ${ }^{1}$, Shi Chen ${ }^{1}$, Hao Hao ${ }^{1}$, Hui Wang ${ }^{1}$, Dan-Feng Pan ${ }^{1}$, Xue-Cou Tu ${ }^{1,2}$, La-Bao Zhang ${ }^{1,2}$, Xiao-Qing $\mathrm{Jia}^{1,2}$, Jian Chen ${ }^{1,2}$, Lin Kang ${ }^{1,2 \dagger}$, and Pei-Heng $\mathrm{Wu}^{1,2}$

1. Research Institute of Superconductor Electronics (RISE), School of Electronic Science and Engineering, Nanjing University, Nanjing, Jiangsu 210023, China

2. Purple Mountain Laboratories, Nanjing, Jiangsu 211111, China

3. School of Physics and Electronic Electrical Engineering, Huaiyin Normal University, Huai'an, Jiangsu, 223300, China.

*qyzhao@nju.edu.cn, †kanglin@nju.edu.cn

\section{Fabrication details}

A $10 \mathrm{~nm}$-thick $\mathrm{NbN}$ film was deposited on a double-side oxidized silicon substrate via reactive DC magnetron sputtering in an $\mathrm{Ar}$ and $\mathrm{N}_{2}$ atmosphere. The $\mathrm{NbN}$ film was patterned into nanowires using electron-beam lithography (EBL) with positive-tone polymethyl methacrylate (PMMA A2), followed by reactive ion etching with $\mathrm{CF}_{4} / \mathrm{Ar}$ plasma. The electrode pads were formed using ultraviolet lithography with a photoresist (AZ1500) and lift-off by depositing $20 \mathrm{~nm} \mathrm{Ti} / 150 \mathrm{~nm} \mathrm{Au}$.

\section{Latched and unlatched operation}

The encoder has two operation modes: (1) latched mode and (2) self-resetting mode. The device operates in latched mode when the channel has a high bias. As shown in Fig. S1a, the corresponding output signal (pulse width $\sim 15 \mathrm{~ns}$ ) is synchronized with the first input signal (pulse width $=5 \mathrm{~ns}$ ) in one bias period. In this mode, the encoder can achieve a high signal-to-noise ratio for the output, a high current gain and better 
driving capability; however, it has a long recovery time and cannot operate under DC bias mode. This mode is suitable for interfacing between nTrons and high-impedance devices, but it is not suitable for high-speed operation.

When the encoder has a low bias, slightly above or below the self-heating current, the encoder does not latch and goes to the self-resetting mode. The low bias regime results in a weaker output signal but allows for high speed operation. The encoder can quickly recover by itself without resetting the bias. As shown in Fig. S1b, successive pulses are triggered by input signals (pulse width $=5 \mathrm{~ns}$ ) for a constant bias. An alternative way to achieve the unlatch operation is using a large inductance in series with the nTron channel. However, this increases the recovery time for the bias current and is not good for achieving fast operation.
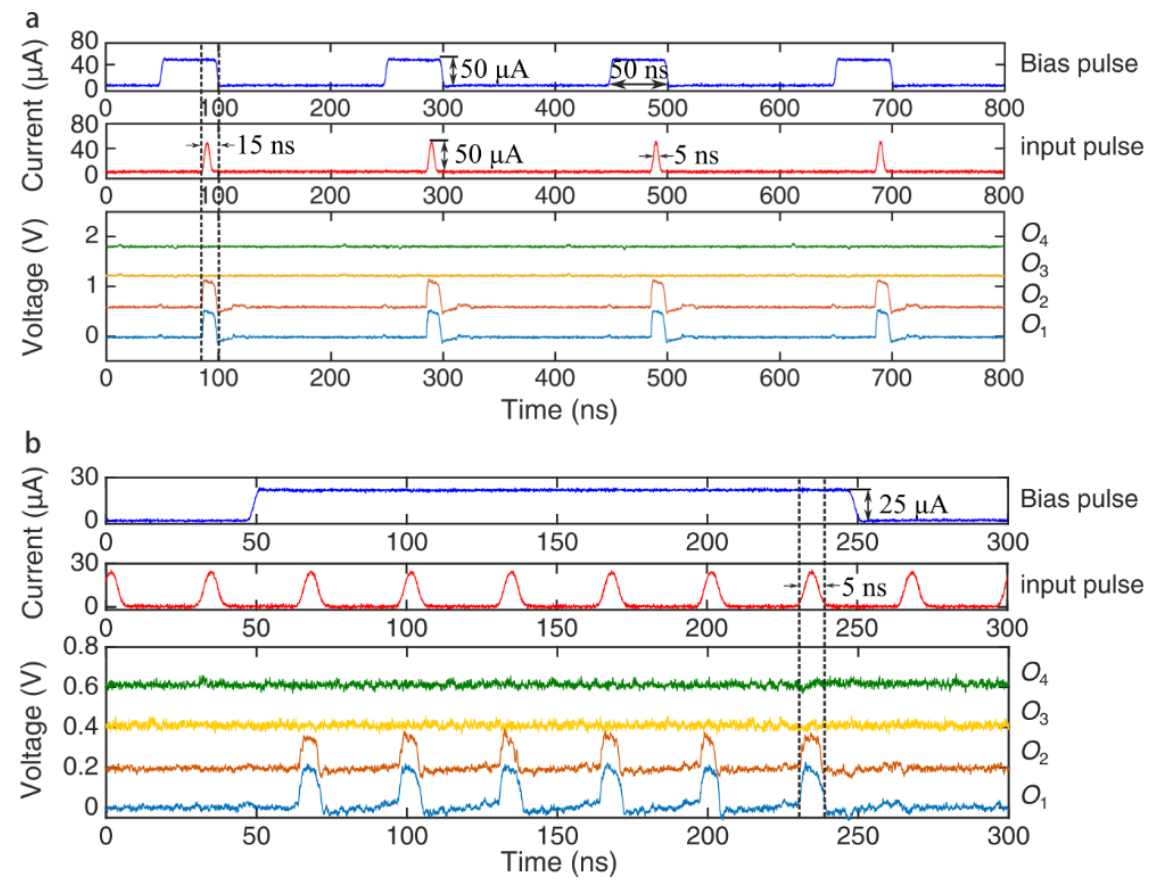

Figure S1. Waveforms for operating the encoder in latched mode (a) and self-resetting mode (b). The input channel is $I_{3}$. The output waveforms $\left(O_{1}\right.$ to $\left.O_{4}\right)$ are shifted along the y-axis for better visualization. 


\section{Characterization of the two-stage nTron amplifier}

To trigger the nTron encoder, the input current had to exceed the critical value. A typical output from an SNSPD was not able to trigger the nTron encoder. Thus, we designed a two-stage nTron amplifier to interface between the SNSND and encoder. The nTrons were patterned from an $\sim 10 \mathrm{~nm}$ thick NbN film. Fig. S2a and Fig. S2b show SEM images of the nTrons. From the SEM measurements, the first nTron had a $49 \mathrm{~nm}$ wide gate and $125 \mathrm{~nm}$ wide channel, while the second $\mathrm{nTron}$ had an $80 \mathrm{~nm}$ wide gate and a $307 \mathrm{~nm}$ wide channel. The critical current of the first nTron was $35 \mu \mathrm{A}$, and it was $106 \mu \mathrm{A}$ for the second nTron. The gate critical current of the nTron gate could not be measured since it was connected with a coupling circuit. Assuming the critical current is linearly dependent on the nanowire width, the estimated critical current of the gate of the first nTron was $14 \mu \mathrm{A}$, and it was $28 \mu \mathrm{A}$ for the second nTron.

a

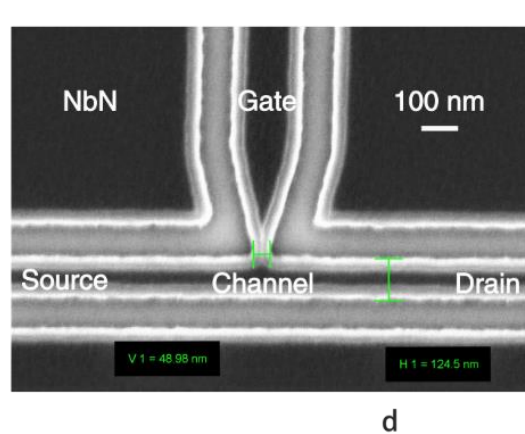

C

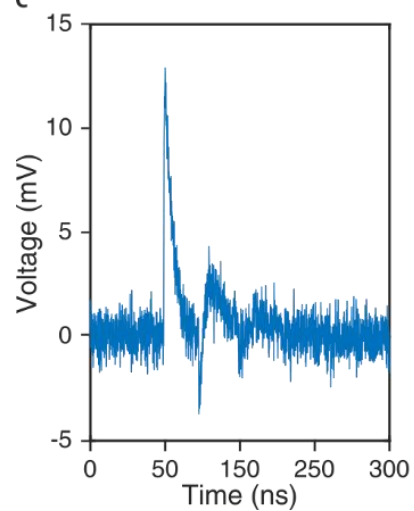

d

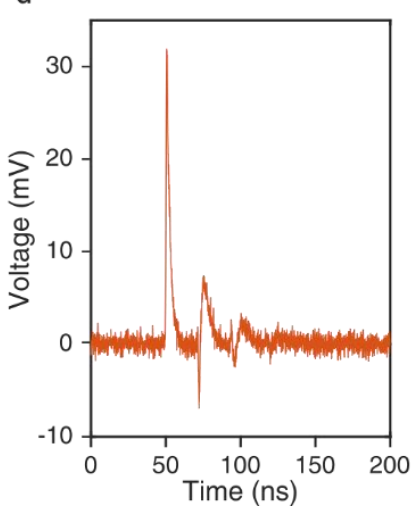

b

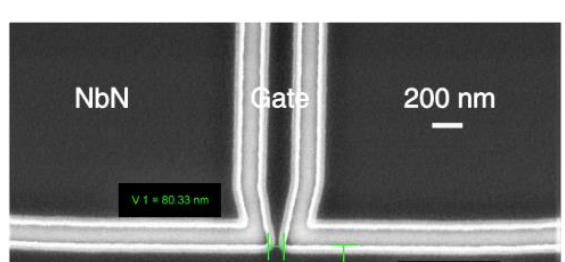

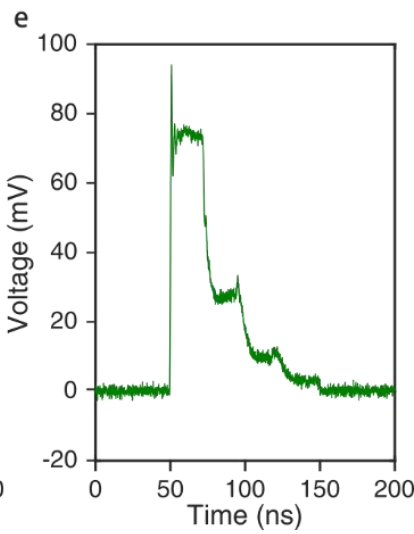

Figure S2. Scanning electron microscope image of the nTrons for building the two-stage nTron amplifier. (a) is the nTron in the first amplifier stage and (b) is the nTron in the second amplifier 
stage. (c), (d) and (e) are waveforms from the SNSPD, the first stage nTron amplifier and the second stage nTron amplifier, respectively. The small pulses after the first peak are the reflections of the room temperature amplifier. In (e), the nTron latched at the beginning but was reset due to negative reflected pulses that lowered the current through the nTron channel.

Fig. S2c shows the output waveforms. We used a room temperature amplifier (RFbay LNA-2500, $25 \mathrm{~dB}$ gain) to read them with an oscilloscope. The SNSPD was biased at $18 \mu \mathrm{A}$. The peak value of the photon detection pulse was $12 \mathrm{mV}$. Assuming a $50 \Omega$ load, the converted output current was $14 \mu \mathrm{A}$. After the first-stage nTron amplifier, the output pulse shown in Fig. S2d increased to $33 \mathrm{mV}$, corresponding to an output current of $37 \mu \mathrm{A}$. Thus, we obtained a current gain of $2.6(8.3 \mathrm{~dB})$. After connecting the second nTron amplifier in series with the first nTron amplifier, the output pulse shown in Fig. S2e increased to $95 \mathrm{mV}$, corresponding to an output current of $107 \mu \mathrm{A}$. It was noticeable that the output pulse from the second nTron amplifier showed a plateau as this nTron was operated close to the latch regime. The total current gain was then $107 \mu \mathrm{A} / 14 \mu \mathrm{A}=7.6(17.6 \mathrm{~dB})$.

\section{Waveforms for 15 photon detections read out by the nTron encoder}

In our experiments, the SNSPD array, nTron amplifier and nTron encoder were fabricated on different chips. To characterize the full operation of the array required many cables; we chose only four detectors to demonstrate the operation principle. However, we connected the output from a single SNSPD to all the input ports of the nTron encoder through a two-stage nTron amplifier and demonstrated correct operations for all the inputs. 
The output waveforms are shown in Fig. S3. We irradiated the SNSPD array with photons by using a 1550 -nm wavelength pulsed laser with a 100 -fs pulse width. The light power was attenuated to the single photon level. A current pulse was used for biasing the nTron channel. The pulse amplitude was $I_{\text {bias }}=45 \mu \mathrm{A}$. We synchronized the nTron bias pulse and the laser pulse so that the onset of the encoder output pulse was triggered by the SNSPD output pulse. The nTron operated in latched mode and was reset by an AC bias pulse. 

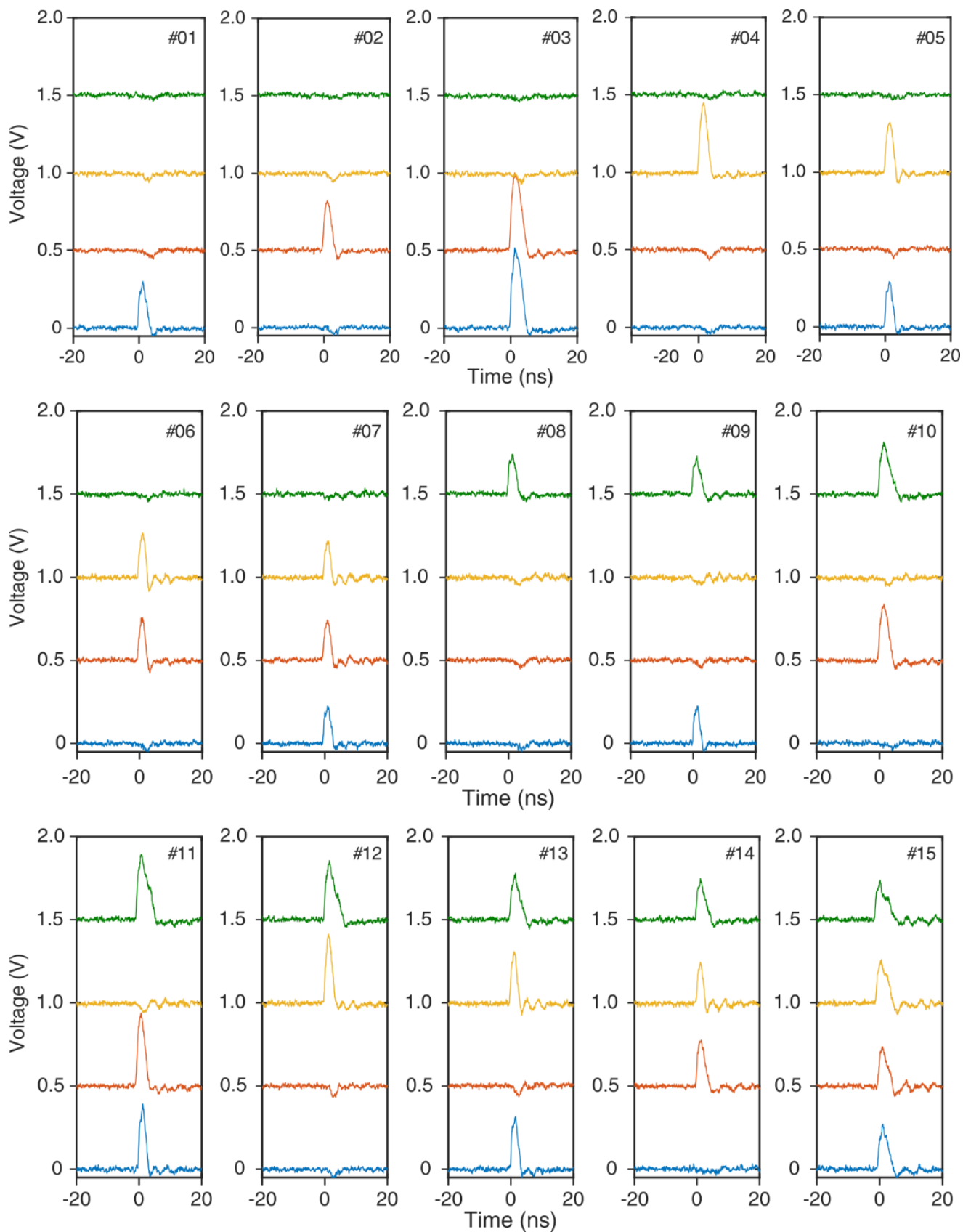

Figure S3. Output waveforms from the encoder for all 15 input ports triggered by the amplified output from an SNSPD. The waveforms are shifted along the $y$-axis for better visualization.

\section{Comparison between the nTron encoder and the SFQ circuit regarding reading SNSPD arrays}




\begin{tabular}{|c|c|c|c|c|c|c|c|}
\hline & $\begin{array}{l}\text { Number } \\
\text { of gates or } \\
\text { junctions }\end{array}$ & $\begin{array}{c}\text { Number } \\
\text { of } \\
\text { SNSPDs }\end{array}$ & Area & $\begin{array}{c}\text { Bias } \\
\text { margin }\end{array}$ & Speed & $\begin{array}{c}\text { Power } \\
\text { dissipation }\end{array}$ & Jitter \\
\hline $\begin{array}{c}\text { nTron } \\
\text { encoder } \\
\text { (our work) }\end{array}$ & 32 & 15 & $\begin{array}{c}4 \times 0.5 \\
\mu \mathrm{m}^{2}\end{array}$ & $18.9 \%$ & $\begin{array}{c}250 \\
\mathrm{MHz}\end{array}$ & $361 \mathrm{nW}$ & $\begin{array}{c}\text { Std value } \\
75 \mathrm{ps} \\
\text { (average) } \\
33 \mathrm{ps} \\
\text { (minimum) }\end{array}$ \\
\hline $\begin{array}{c}\text { SFQ } \\
\text { readout } \\
\text { circuits* }\end{array}$ & 2610 & 64 & $\begin{array}{l}\sim 10 \\
\mathrm{~mm}^{2}\end{array}$ & $\begin{array}{l}-10 \% \sim \\
+20 \%\end{array}$ & $\begin{array}{l}12.5 \\
\mathrm{MHz}\end{array}$ & $250 \mu \mathrm{W}$ & $\begin{array}{c}\text { (FWHM) } \\
56.5 \mathrm{ps} \\
\text { (read out } \\
\text { SSPD \#16) }\end{array}$ \\
\hline
\end{tabular}

* S. Miyajima, M. Yabuno, S. Miki, T. Yamashita, and H. Terai, "High-time-resolved 64-channel single-flux quantum-based address encoder integrated with a multi-pixel superconducting nanowire single-photon detector," Opt. Express 26(22), 29045 (2018). 